\title{
Estimation of Insulating Materials Depreciation and Forecasting the Residual Cable Resource Considering the Current Core Temperature
}

\author{
Marina N. Dubyago and Nikolay K. Poluyanovich
}

\begin{abstract}
At the design stage of power cable lines (PCL), according to the methodology in IEC 60287, its throughput is determined, taking into account all possible factors arising from the exploitation- select the coefficients that provide the reserve for heating, so in some cases the cables are under loaded, and sometimes operate at the limit of thermal stability. Currently, the technology of measuring cable distributed temperature is actively introduced, aimed at solving these problems and allowing to control the surface temperature of the cable in real time over the entire length of the PCL. Low thermal conductivity of cable insulation leads to a high temperature gradient, so the temperature of the most heated section in the cable section (near the core) is significantly different from the measured temperature, and in transient modes this difference can increase several times. Therefore, the problem of creating mathematical models and algorithms for estimating the transmission capacity, calculation and prediction of the PCL core temperature in real time on the basis of the data of the temperature monitoring system, taking into account the change in the current load of the line and the external conditions of the heat sink, is urgent.
\end{abstract}

Index Terms-Insulating materials, forecasting, thermal processes, power supply systems.

\section{INTRODUCTION}

Estimating the throughput of PCL. The tasks of continuous monitoring of PCL thermal regime are [1], [2]: • determination of the cable temperature in time and place along the PCL route; • timely prevention of PCL current overloads; - fore-casting the permissible load when the cable reaches the maximum operating temperature; • Creation of optimum PCL current loads, that ensuring continuity of power supply to consumers and reducing the probability of occurrence of emergency events. Therefore, the problem of creating mathematical models and algorithms for estimating the transmission capacity, calculation and prediction of the PCL core temperature in real time on the basis of the data of the temperature monitoring system, taking into account the change in the current load of the line and the external conditions of the heat sink, is urgent.

\section{A. Analysis of Methods for the Thermal Field Calculating}

The thermal field for the PCL, whose construction is shown in Fig. 1, is described by the differential heat equation (1), which determines the change in the

Manuscript received September 17, 2018; revised January 12, 2019.

The authors are with Southern Federal University, Russia (e-mail: nik158@mail.ru,w_m88@mail.ru). temperature distribution in the volume as a function of time.

$$
\frac{\partial T}{\partial t}=\operatorname{div}(\chi \cdot \operatorname{grad}(T))+{ }_{c_{v}}^{q_{v}}
$$

where, $T$ - temperature, ${ }^{\circ} \mathrm{K} ; t$ - time, s; $q_{v}$ - capacity of volumetric heat sources (volumetric heat dissipation density), $\mathrm{W} / \mathrm{m}^{3} ; c_{\nu}$ - the volume heat capacity, $\mathrm{J} /(\mathrm{K} \cdot \mathrm{m} 3) ; \chi$ - thermal diffusivity, $\mathrm{m}^{2} / \mathrm{s}$. The coefficient $\chi$ is determined by the formula $V c=\lambda c$, where $\lambda$ is the thermal conductivity coefficient, $\mathrm{W} /(\mathrm{m} \bullet \mathrm{K})$. a) b)

The solution of equation (1) in statics can be obtained for cables having an axisymmetric construction. At the heart of the methodologies for PCL thermal steady-state regimes estimating described in the standard IEC 60287 (1989) is the Neuer-McGrath model, which reduces to determining the permissible load current of the cables

$$
I_{\text {add }}=\sqrt{\frac{T_{a d d}-T_{0}-Q_{d}\left(0.5 R_{T 1}+R_{T 2}+R_{T 3}+R_{T 4}\right)}{R_{c}\left(R_{T 1}+\left(1+\Delta_{1}\right) R_{\mathrm{T} 2}+\left(1+\Delta_{1}+\Delta_{2}\right)\left(R_{\mathrm{T} 3}+R_{\mathrm{T} 4}\right)\right.}}
$$

where $T_{\text {add }}$ - permissible cable working temperature (for CLP insulation $-90^{\circ} \mathrm{C}$ ), ${ }^{\circ} \mathrm{C} ; T_{0}$ - ambient temperature, ${ }^{\circ} \mathrm{C} ; Q_{d}$ - the dielectric loss in insulation per unit length, W/m; $R_{c}$ electrical resistance of the core to alternating current per unit length at the maximum operating temperature, $\mathrm{Ohm} / \mathrm{m}$; $R_{T 1}, R_{T 2}, R_{T 3}, R_{T 4}$ - thermal resistances of various layers of the cable and environment design per unit length, ${ }^{\circ} \mathrm{K} \cdot \mathrm{m} / \mathrm{W}$; $\Delta_{1}, \Delta_{2}$ - the ratio of total losses in the screen and armor to the sum of losses in the conductor.

Due to the large number of coefficients and formulas used in the IEC 60287 standard, its application for capacity estimation is a complex task. Therefore, they suggest using the COMSOL Metaphysics software complex, based on the finite element method application. The program allows to take into account the PCL design, solve non-linear problems, build multi-physical models that connect the electric current with the heat dissipation in the cables, taking into account the electromagnetic effects (for example, the joint calculation of the thermal and electromagnetic fields). In [3], [4], it is proposed to calculate only the thermal resistance of the soil, and to determine the allowable current using IEC 60287. In [5], on the contrary, IEC 60287 is used to determine the values of internal heat sources, and the temperature of the cables is calculated using field models.

\section{B. PCL Throughput}

In [6] they solve the problem of determining the PCL throughput, laid in the channel, with a laminar incompressible air flow, taking into account natural 
convection in the ELCUT program. In [4], the authors consider the influence of the heat path, crossing and passing along the PCL in the ground. In [7], the problem of cables temperature estimating is solved as follows. The heat release is determined by the calculation of the electromagnetic field on the basis of the differential equation (3) written with respect to the z-component of the complex vector magnetic potential:

$$
\frac{\partial}{\partial x}\left(\frac{1}{\mu} \frac{\partial \dot{A}_{z}}{\partial x}\right)+\frac{\partial}{\partial y}\left(\frac{1}{\mu} \frac{\partial \hat{A}_{z}}{\partial y}\right)=-\delta_{e x}+j \omega \gamma \hat{A}_{z}
$$

where $x, y, z$ are the coordinates of the Cartesian plane, $\mathrm{m} ; \mu$ - absolute magnetic permeability, $\mathrm{F} / \mathrm{m} ; A_{z}$ - the $z$ component of the complex vector magnetic potential; $\delta_{e x}$ - complex density of the external current, $\mathrm{A} / \mathrm{m} ; j$ - the imaginary unit; $\omega$ - the angular frequency, $\mathrm{rad} / \mathrm{s} ; \gamma$ - the specific electrical conductivity, $\mathrm{S} / \mathrm{m}$.

Then, based on the heat flux found in the PCL design, the total thermal field is determined taking into account convective heat transfer, thermal conductivity and radiation energy, depending on the method of cable laying. But this approach initially contains an error, since at the first moment of the electromagnetic field calculation, conductors temperature is not known, which does not allow to correctly consider the effect of temperature on the resistance of the conductors.

Thus, in [4], [5], [7], [8], the mutual influence of the thermal and electromagnetic fields on each other is not fully considered, which reduces the accuracy of the calculations. The models described in [3], [5] take into account the largest number of factors affecting the PCL capacity, and allow calculations to be made in dynamics, but in this case, high processor performance and a large amount of computer memory are required to reduce computation time. The use of the models in the monitoring system is impossible because the finite element method (FEM) requires a large amount of computer resources, which is inadmissible in real-time calculations.

\section{FORECASTING THE PCL CORE TEMPERATURE}

In addition to capacity assessing to monitor thermal conditions, the evaluation of the core temperature in real time and the timely prevention of current PCL overloads [2] include the forecasting of cable veins temperature in real time. The latter means that it is necessary to constantly predict the cables heating for a while forward, and such that it is possible to take measures to reduce the CL load. Obtaining an analytical solution of equation (1) in dynamics is possible under certain assumptions, or for simple special cases. When calculating the heating of a single-phase single cable [9] lay in the ground, the ground surface was considered isothermal, and the cable was a thin-walled tube of radius $r_{c}$. For the boundary between the cable and the ground, the heat balance equation was written:

$$
-2 \pi \lambda_{s} r_{c}(\partial T / \partial r)=Q_{c}
$$

where $Q_{c}$ - cable thermal losses, W/m; $\lambda_{s}$ - coefficient of soil thermal conductivity, $\mathrm{W} /(\mathrm{m} \cdot \mathrm{K})$.
Equations (1) and (4) in [9] are solved by the operator method, applying the Corson-Heaviside transform to them. To calculate the temperature at an arbitrary point in the soil, the following formula was obtained:

$$
T(t)=\frac{Q_{c}}{2 \pi \lambda_{s}}\left\lceil\ln \frac{d \prime}{d}-\int_{0}^{\infty} \frac{J_{0}(x)-J_{0}\left(\frac{d^{\prime}}{d} x\right)}{x} e^{-\frac{a}{d^{2}} x^{2} t} d x\right\rceil
$$

where $d$ and $d^{\prime}$ are the distances from the considered point to the cable center and to the its mirror image center, respectively, m; $J_{0}$ - a cylindrical function of the first kind of zero order.

If instead of d substitute the cable radius rk and calculate the corresponding distance to the image, we get a formula for the cable surface temperature. But to assess the thermal mode of the cable, you need to know the core temperature, which will differ significantly from the surface temperature due to the low thermal conductivity of the polyethylene.

In [10], equation (1), it was decided to determine the temperature on the cable surface by means of the integral exponential function, because in them the cable was considered an infinitely thin linear heat source.

$$
-E i(-x)=\int_{0}^{\infty} \frac{e^{-v}}{v} d v
$$

The formula for the surface temperature of a single cable laid at a depth $h$ in the ground is:

$$
T_{c}(t)=T_{0}+Q_{k} \frac{1}{2 \pi \lambda_{s}}\left[-E i\left(-\frac{D_{c}^{2}}{16 a t}\right)+E i\left(-\frac{h^{2}}{a t}\right)\right]
$$

where $D_{c}$ - the outer cable diameter, $\mathrm{m}$.

The temperature increase in the cable $m$ under the action of the cable $\mathrm{n}$ near it is predetermined by the formula:

$$
T_{m n}(t)=Q_{n} \frac{1}{2 \pi \lambda_{s}}\left[-E i\left(-\frac{d_{m n}^{2}}{4 a t}\right)+E i\left(-\frac{d^{\prime}{ }_{m n}^{2}}{4 a t}\right)\right]
$$

where $Q_{n}$ - thermal losses in the cable $\mathrm{n}, \mathrm{W} / \mathrm{m} ; d_{m n}$ and $d^{\prime}{ }_{m n}$ - the distances from the center of the cable $m$ to the center of the cable $\mathrm{n}$ and its mirror image, respectively, $\mathrm{m}$.

But, this solution also does not allow to determine the change in the temperature of the cable core.

In [11], the author proposed a formula for calculating the core temperature based on the boundary conditions shown in Fig. 1, representing the cable itself, one part, and the environment (soil) - the second. The core temperature in the dynamics looks like this:

$$
\begin{aligned}
& T_{c}(t)=\frac{2 Q_{c}}{\pi^{3 \lambda_{s}}}=\int_{0}^{\infty} \frac{1}{1} \frac{\left.\phi_{1} N_{2}\right)^{2}\left(1-e^{-\frac{a \phi^{2}}{r_{c}^{2}} t}\right)}{\phi^{3}} \\
& d \phi
\end{aligned}
$$

Here $\varphi$ - the integration variable; $J_{0}, J_{1}$ - cylindrical functions of the first kind, zero and first order, respectively, $Y_{0}$ and $Y_{1}$ - cylindrical functions of the second kind, of zero and first order, respectively; The functions $F_{0}(\varphi)$ and $F_{1}(\varphi)$ 
denote the following expressions:

$$
\begin{gathered}
F_{0}(\phi)=M_{1} M_{2} N_{2} \phi^{3}-\left(M_{1}+M_{2}\right) N_{1} N_{2} \phi \\
F_{1}(\phi)=M_{1} M_{2} \phi^{4}-\left(M_{1} N_{2}+M_{2} N_{1}+M_{1} N_{1}\right) \phi^{2}+N_{1} N_{2}
\end{gathered}
$$

where

$$
\begin{aligned}
M_{1}=\frac{\mathrm{C}_{1}}{2 \pi \mathrm{c}_{V} r_{k}^{2}}, M_{2} & =\frac{\mathrm{C}_{2}}{2 \pi \mathrm{c}_{V} r_{k}^{2}}, N_{1}=\frac{1}{2 \pi \lambda R_{t 1}} \\
N_{2} & =\frac{1}{2 \pi \lambda R_{t 2}}
\end{aligned}
$$

\section{The LAYered MOdel of CABle Temperature DISTRIBUTION}

\section{A. Analysis of Thermal Processes by Layers}

A mathematical model was developed (12) [12], which makes possible to calculate the temperature distribution over the layers (Fig. 1), and a formula is derived that makes it possible to determine $\theta^{\circ}$ in the core.

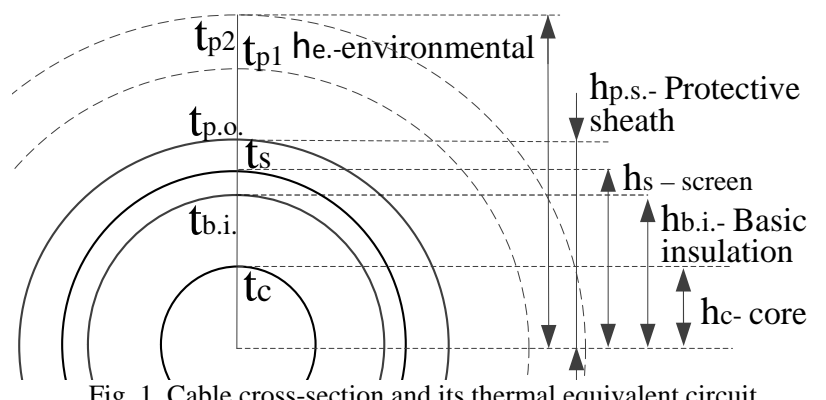

Fig. 1. Cable cross-section and its thermal equivalent circuit.

The temperature profile in the section of the power cable was determined by a system of equations, in ac-cordance with the theory of thermal conductivity, Fig. 2, where $I_{C}, I_{S}$ - currents on the core and the screen; $\varkappa$ - coefficient of thermal conductivity; $j_{C}=I_{C} S_{C} ; j_{S} C=I_{S} / S_{S} ; S_{C}, S_{S}$ - crosssectional of the core and screen surface areas; $\gamma_{C}, \gamma_{S}-$ conductivity of the core and the screen.

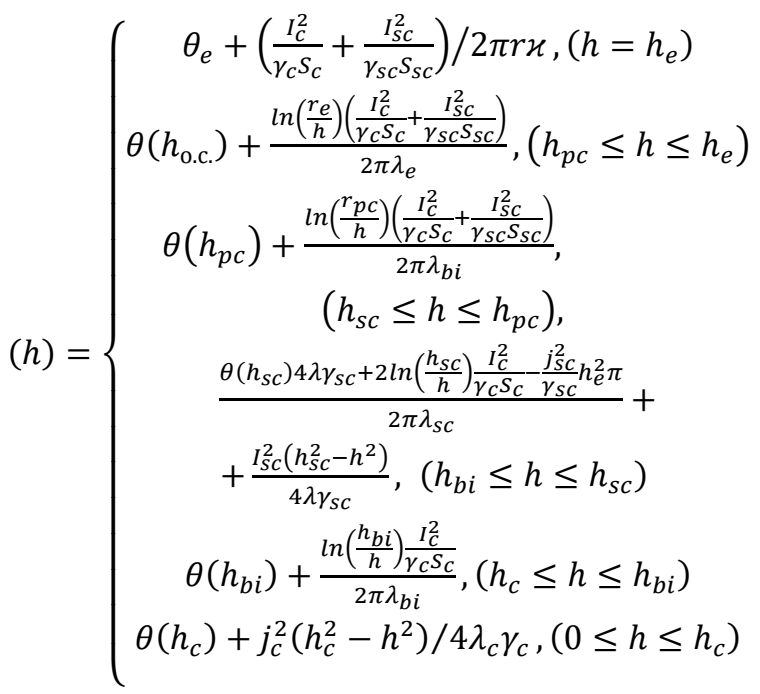

In accordance with the equations system (12), MatLab constructs a temperature profile in the cable cross section and the environment, Fig. 2 Calculations performed at a current in the cable core $640 \mathrm{~A}$. The current in the cable sheath is determined by operating cable mode (current in the cable core). The current in the cable casing was adopted as a percentage of the current in the cable core $0 \%, 20 \%$, $40 \%$ and $60 \%$, Fig. 2(b).

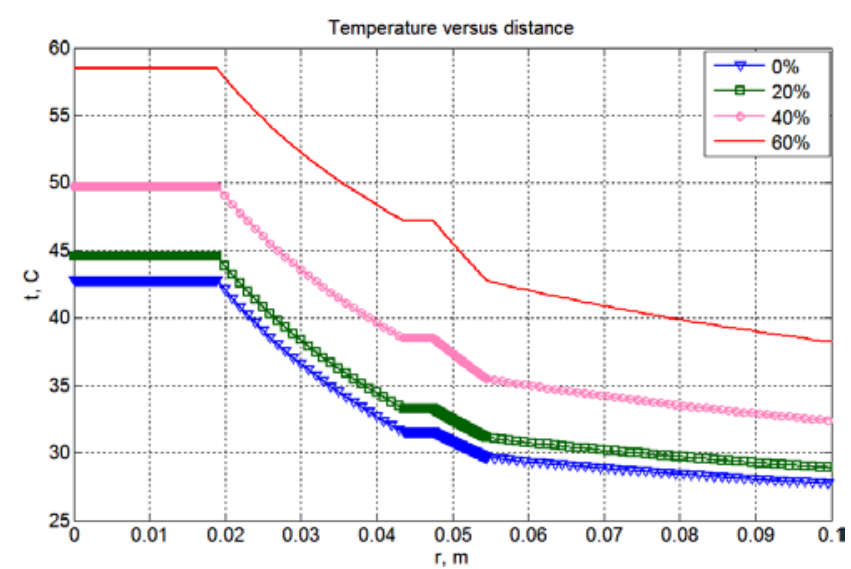

(a)

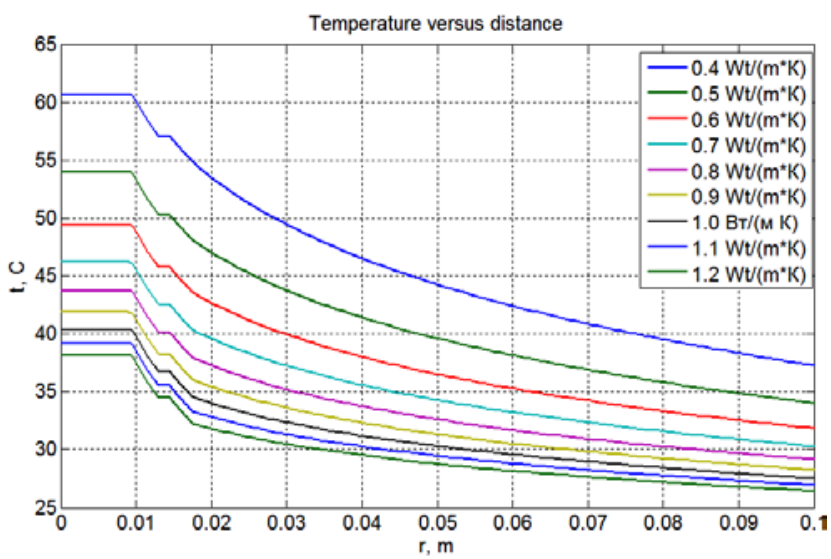

(b)

Fig. 2. Temperature distribution graphs at the characteristic points of the CL cross section: (a) - at different percentages of the current screen on the current conductor; (b) - for various thermal conductivities of the soil.

\section{B. Calculation of the Cable Core Temperature}

The calculation method for determining the temperature of the PCL core hotter point, using the measured values of the temperature of its surface, the ambient temperature, and the core currents, according to the following formula [13, p.526].

An analytical dependence (13) is proposed for determining the temperature of the most heated insulation point (cable core) obtained from the system (12) for calculating the cable core temperature taking into account the surface temperature of the cable, the surrounding medium and the core current:

$$
\theta_{c}(t)=\theta_{p c}(t)+\frac{n \cdot I^{2} \cdot \rho_{20} \cdot m \cdot T_{c} \cdot K_{p}\left[1+\alpha \cdot\left(\theta_{a d d}-\theta_{e}(t)\right)\right]}{S}
$$

where $\theta_{c}$ - calculated temperature of the cable core, ${ }^{\circ} \mathrm{C} ; \theta_{p c}$ $=23^{\circ} \mathrm{C}$ - measured temperature of the cable surface (protective casing); $n=1$ - number of wires; $I$ - the maximum cable current when measuring, $\mathrm{A} ; \mathrm{p}_{20}=2.8 \cdot 10-8$ $\mathrm{Ohm} \cdot \mathrm{m}$ - specific electrical resistance of the cable core at 
$20^{\circ} \mathrm{C} ; T_{c}=0.0028^{\circ} \mathrm{C}-\mathrm{m} / \mathrm{W}$ - the sum of thermal resistances of insulation and protective covers of the cable; $\mathrm{m}$ is the experimental factor; $K_{p}=1.02$ is the correction coefficient for bringing the electrical resistance to the design temperature [14]; $\alpha=0,0043081 /{ }^{\circ} \mathrm{C}$ - temperature coefficient of resistance of the vein material; $\theta_{\text {add }}=90{ }^{\circ} \mathrm{C}$ long-term permissible core insulation temperature; $\theta_{e}=$ $23{ }^{\circ} \mathrm{C}$ - measured environment temperature; $S=0.0038465$ cross-section of the cable core, $\mathrm{m}^{2}$.

Equations (13) take into account the stationary thermal processes in the cable and are valid for steady-state values of the measured quantities and makes it possible to calculate the cable core temperature as simply as possible. During operation, the measured values change over time, so to account for them it is necessary to calculate the nonstationary thermal processes.

\section{EXPERIMENTAL STUDIES}

To test the developed mathematical model adequacy, a powerful step-down transformer was used, which enabled to obtain a current of $640 \mathrm{~A}$, and a measuring system was collected to study thermal processes in single-core cables.

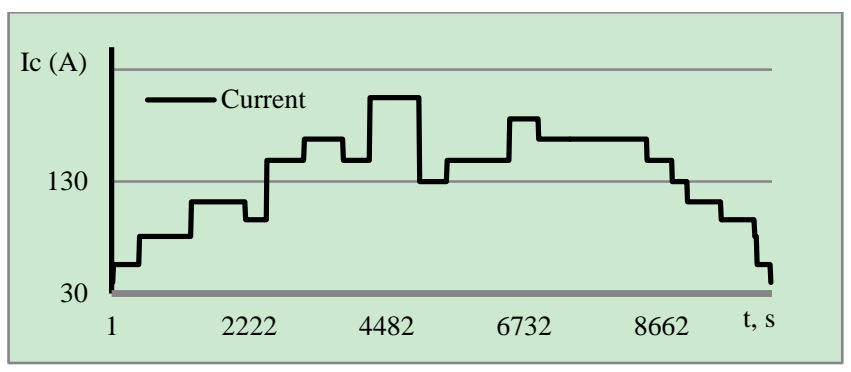

Fig. 3. Experimental dependence for the PCL current.

The temperature sensors were layer-by-layer located inside and on the PCL surface, as well as at some distance from it. As a sample we used segments of known cables with XLPE insulation PUv-1x30 / 25-10 with a length of $0.85 \mathrm{~m}$. Figure 4 shows experimental and calculated time diagrams of cable temperature $\mathrm{APv} \mathrm{Pu}-1 \mathrm{x} 30$ / 25-10 in volume same experience as Fig. 3.

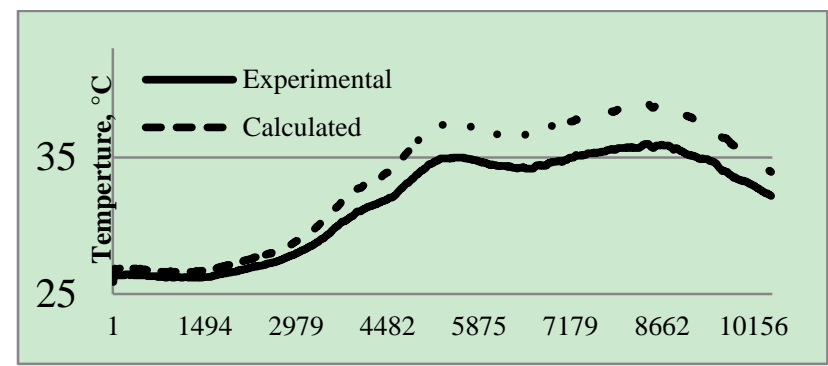

Fig. 4. Experimental and calculated time diagrams cable temperature APv $\mathrm{Pu}-1 \times 30 / 25-10$.

TABLE I: THE VALUES OF THE QUALITY INDICATORS OF THE THERMAL

\begin{tabular}{|c|l|c|}
\hline \multicolumn{3}{|c|}{ MODEL } \\
$\begin{array}{c}\text { № } \\
\text { p.p. }\end{array}$ & \multicolumn{1}{|c|}{$\begin{array}{c}\text { Calculation accuracy } \\
\text { indicators }\end{array}$} & $\begin{array}{c}\text { The errors in the calculation } \\
\text { result }\end{array}$ \\
\hline 1 & Range of accuracy $\Delta \theta_{\mathrm{MAX}}$ & 2,9 \\
\hline 2 & Average vectors value & 1,8 \\
\hline 3 & Coefficient of correlation, $\mathrm{r}$ & 0,999 \\
\hline 4 & Maximum relative error, $\%$ & 5,4 \\
\hline
\end{tabular}

The results of calculating the quality parameters of the mathematical model from the experiment, obtained by comparing the measured and calculated cable temperature, are given in Table I.

Thus. the maximum absolute error in the temperature calculating, in comparison with the directly measured temperature, did not exceed $2.9{ }^{\circ} \mathrm{C}$. The obtained high values of the correlation coefficient, equal to 0.999 , indicate sufficient adequacy of the mathematical model to real thermal processes. The proposed analytical dependence can be used as a basis for calculating the PCL thermal processes in real time mode, as its correspondence is supported by experimental data. The obtained diagrams show an obvious correlation between the experimental and calculated temperature curves, which indicates the correct direction when choosing a mathematical model [15].

\section{EVALUATION OF INSULATION DEPRECIATION TAKING INTO ACCOUNT THE CURRENT CORE TEMPERATURE}

\section{A. Thermochemical Aging}

One of the tasks of PCL diagnosing is to estimate the depreciation and the remaining life of the cable insulation. When the temperature exceeds the nominal temperature, chemical reactions occur in the insulation and accelerate, which leads to its properties deterioration and an increase in the tangent of the dielectric loss angle. The latter leads to an increase in thermal losses and the core temperature. These processes are called thermo chemical aging and lead to an unacceptable state of isolation in terms of electrical strength. The rate of these processes follows the Arrhenius law. The time of destruction of insulation is determined from the dependence [16].

$$
t=A e^{\frac{W a}{K T}}
$$

where A - an adjustable constant; Wa - the activation energy of the aging process, $\mathrm{J}$; $\mathrm{k}$ - the Boltzmann constant, $\mathrm{J} / \mathrm{K}$.

There are many different mathematical models that allow predicting the destruction of insulation under given operating conditions. According to [17], it is possible to determine the time for the development of polymer insulation (service life, tsl) at the operating temperature and electric field strength using formula

$$
t_{s l}=\frac{h_{P l}}{k T} e^{\frac{\Delta H-T \Delta S}{k T}} \operatorname{csch}\left(q_{e} \lambda_{a} \frac{E}{k T}\right)
$$

where $h_{P l}$ - the constant $\mathrm{J} \cdot \mathrm{s}$ stripe, $\Delta H$ - the enthalpy of activation, $\mathrm{J} ; \Delta S$ - entropy of activation, $\mathrm{J} / \mathrm{K} ; q_{e}$ - the elementary charge, Кл; $\lambda a$ - length of activation, m; $E$ electric field strength, $\mathrm{V} / \mathrm{m}$.

For large values of temperature and strength, it is proposed in [17] to use the strengthened formula

$$
t_{s l}=\frac{h_{P l}}{k T} e^{\frac{\Delta H-T \Delta S-q_{e} \lambda_{a} E}{k T}}
$$

It can be seen from expressions (15), (16) that the service life of the insulation is in a complex dependence on temperature. The assumption in (15), (16) that the 
parameters of the insulation state are unchanged during the operation of the cable, does not correspond in real operating conditions. Due to the change in ambient temperature, the time of year and the load current, the core temperature during the normal operation of the cable can vary considerably. At a variable temperature, the aging effect is added together. Therefore, in order to quantify the reduction in the service life of SCR, it is necessary to calculate the wear of insulation at each value of the core temperature (Fig. 4) that occurs during operation, and then add them.

\section{B. Forecasting Thermal Insulation Wear}

In [12] one of the possible algorithms for calculating the relative wear of insulation was proposed. However, it does not take into account the thermal transition in the cable, and the core temperature is suggested to be calculated from the ratio of the current current to the nominal value. With the help of the developed algorithm for calculating the core temperature in real time, it is possible to more accurately estimate the thermal wear of the insulation, since the actual core temperature at each instant of time will be known (Fig. 4).

Then at each step of measuring the screen temperature and calculating the current core temperature, it is necessary to calculate the relative wear of the insulation (Fig. 5). For this purpose, it is proposed in [12] to calculate the relative wear rate of insulation:

$$
V_{i}=\frac{v_{i}}{v_{\text {ном }}}
$$

where $i$ - the number of the current measurement or algorithm calculation step; $v=1 /$ tsl - wear rate of insulation (inverse value of service life), $1 / \mathrm{s} ; v_{\text {ном }}$ - wear rate of insulation at the maximum permissible operating temperature $\left(\right.$ Tnom $\left.=90^{\circ} \mathrm{C}\right), 1 / \mathrm{s}$.

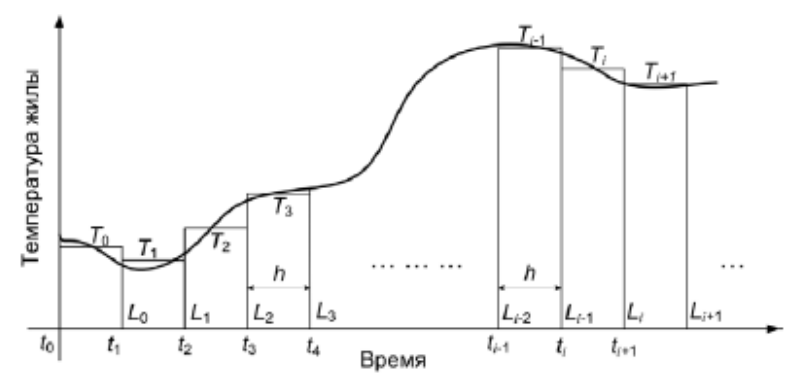

Fig. 5. Real graph and diagram of calculated core temperatures.

If the wear rate is multiplied by the duration of the current temperature $\mathrm{h}$ and divided by the total cable service time $t_{n}$, we obtain the relative wear of the insulation for the time interval between the measurements

$$
L_{h}=\frac{1}{t_{n}} V_{i} h \cdot 100 \%
$$

Using the formulas (15)-(18), it is possible to calculate the new value of the relative wear of the cable insulation after the next measurement of the screen temperature and calculation of the current residence temperature by formula (19) for the operating mode and according to formula (20) for the stress regime, When the values of the parameters have exceeded the allowed values:

$$
\begin{aligned}
L_{i}= & L_{i-1}+\frac{1}{t_{n}} \frac{T_{i}}{T_{\text {rat }}} e^{\frac{\Delta H-T_{\text {rat }} \Delta S}{k T_{\text {ном }}}-\frac{\Delta H-T_{i} \Delta S}{k T_{i}}} \operatorname{sh}\left(q_{e} \lambda_{a} \frac{E}{k T_{i}}\right) \\
& \cdot \operatorname{csch}\left(q_{e} \lambda_{a} \frac{E}{k T_{\text {rat }}}\right) h \cdot 100 \%, \\
L_{i}= & L_{i-1}+\frac{1}{t_{n}} \frac{T_{i}}{T_{\text {rat }}} e^{\frac{\Delta H-T_{\text {rat }} \Delta S-q_{e} \lambda_{a} E}{k T_{\text {rat }}}-\frac{\Delta H-T_{i} \Delta S-q_{e} \lambda_{a} E}{k T_{i}}} \\
& \cdot h \cdot 100 \% .
\end{aligned}
$$

where $L_{i-1}$ - the previous value of the relative wear of insulation, \%; $T_{i}$ - the current value of the core temperature determined by the proposed algorithm.

Thus, it is possible to control the PCL insulation thermal aging in the real-time mode taking into account the vein temperature changing during operation. The algorithm for calculating the cables conductors temperature will allow the degree estimation of PCL insulation thermal aging taking into account the dynamics of the actual core temperature in real time.

\section{ACKNOWLEDGMENT}

This work was supported by Russia's Southern Federal University grant №BнГр-07/2017-15: Development of theoretical foundations and creation methods of intelligent multivariable control systems for generating processes, transportation, distribution and consumption of energy.

\section{REFERENCES}

[1] O. V. Udovichenko, “Temperature monitoring of high voltage cable lines based on cables with XLPE insulation," in Proc. of Power Transmission Lines 2008: Design, Construction, Operation Experience and Scientific and Technical Progress: Materials of the III Russian Scientific-Practical Conference with International Participation - Novosibirsk, pp. 301-304, 2008.

[2] Yu. A. Lavrov, "High voltage cables with XLPE insulation. Requirements for economy, reliability, environmental friendliness," News of Electrical Engineering, no. 2, 2008.

[3] S. Dubitsky, G. Greshnyakov, and N. Korovkin, "Refinement of underground power cable ampacity by multiphysics FEA simulation," International Journal of Energy, no. 9, pp. 12-19, 2015.

[4] V. V. Titkov, S. M. Dudkin, R. D. Tukeev, and A. V. Kosorukov, "The capacity limitations of power transmission cable lines in the structure of civil and industry engineering networks," Magazine of Civil Engineering, no. 6, pp. 75-83, 2014.

[5] G. V. Greshnyakov, G. G. Kovalev, and S. D. Dubnitsky, "On the issue of selecting the maximum permissible currents of power cables," Cables and Wires, no. 6, pp. 12-16, 2011.

[6] E. Yu. Navalikhin, N. M. Trufanov, "Mathematical modeling of the processes of heat and mass transfer in the cable channel and determination of the rational transmit power of cables," in Proc. of Internet-Conference of Applied Mathematics, Mechanics and Control Processes: Materials of All-Russian Scientific-Technical for Students and Young Scientists-Perm, pp. 217-227, 2013.

[7] V. Titkov, S. Dudkin, "Cable lines 6-10 kV and higher, influence of methods of laying on the temperature regime," News of Electrical Engineering, vol. 3, no. 75, 2012.

[8] L. A. Kovrigin, N. A. Belkin, R. A. Biyanov, A. S. Karsakov, and S. R. Shangarayev, "Calculation of temperature fields and current loads of cables in ANSYS,” Cable News, no. 4, pp. 91-95, 2009.

[9] S. D. Cold, "Heating and cooling of the cable laid in the ground," Electricity, no. 6, pp. 35-40, 1964.

[10] L. R. Ingersoll, O. J. Zobel, and A. C. Ingersoll, Heat Conduction with Engineering, Geological and Other Applications, New York: McGraw-Hill, 1954.

[11] F. H. Buller, "Thermal transient on buried cables," AIEE Transactions, vol. 70, pp. 45-55, 1951.

[12] V. M. Baranov. Method for estimating the life of power cables. [Online] http://energo.mblabsoft.com/articles/ResourceOfCables.pdf 
[13] E. F. Makarov, Reference Book on Electrical Networks 0.4-35 kV and 110-1150 kV, Russia: Papyrus PRO, 2004.

[14] M. N. Dubyago, N. K. Poluyanovich, and D. V. Bur'kov, "Development of a method for predicting the aging process of insulation based on the thermoflu-pertuation theory of partial discharges," Engineering Bulletin of the Don, no. 3, 2017.

[15] Electric Cables. Calculation of the Rated Current Load--Part 1-1: Equation for Calculating the Rated Current Load (100\% Load Factor) and Calculating Losses-General Provisions. GOST State Standard no. R MEK 60287-2-1-2009.

[16] S. D. Cold, S. V. Serebryannikov, and M. A. Fighting, Methods of Testing and Diagnostics in Electrical Insulating and Cable Technology: A Textbook, Russia: Publishing house MPEI, 2009, p. 232.

[17] C. Dang, J. L. Parpal, J. P. Crine, "Electrical aging of extruded dielectric cables: Review of existing theories and data," IEEE Transactions on Dielectrics and Electrical Insulation, vol. 3, no. 2, pp. 237-247, 1996.

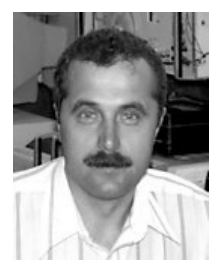

Nikolay K. Poluynovich is Associate Professor of Electrical Engineering and Mechatronics from 1995 to present. And also is an author of more than 250 published works. His phone number is (8634) 37-1694.

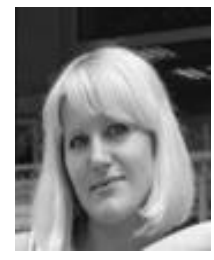

Marina N. Dubyago is graduate student and her specialty is system analysis, management and information processing. Her research interests are renewable energy (development of devices matching the frequency of wind turbines with a common power grid), as well as the development of systems for autodoped isolation control power cable lines. Her phone number is 89281758225 . 